Document downloaded from:

http://hdl.handle.net/10251/60354

This paper must be cited as:

Rodriguez Martinez, A.; Svilainis, L.; V. Dumbrava; Dumbrava, V.; Chaziachmetovas, A.; Salazar Afanador, A. (2014). Automatic simultaneous measurement of phase velocity and thickness in composite plates using iterative deconvolution. NDT and $E$ International. 66:117-127. doi:10.1016/j.ndteint.2014.06.001.

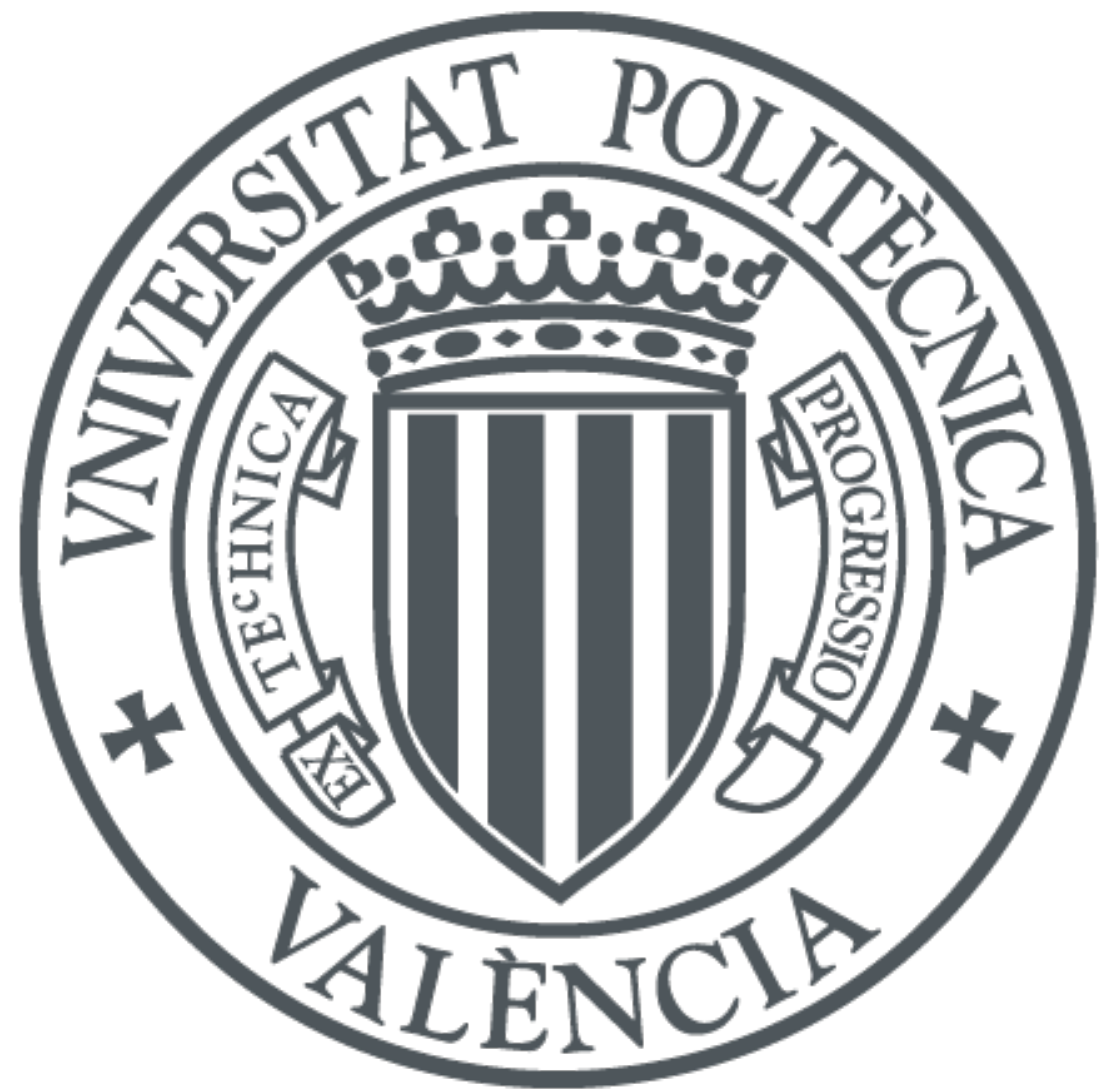

The final publication is available at

http://dx.doi.org/10.1016/j.ndteint.2014.06.001

Copyright Elsevier

Additional Information 


\title{
Automatic Simultaneous Measurement of Phase Velocity and Thickness in Composite Plates Using Iterative Deconvolution
}

\author{
A. Rodríguez ${ }^{\mathrm{a}, *}$, L. Svilainis ${ }^{\mathrm{b}}$, V. Dumbrava ${ }^{\mathrm{b}}$, A. Chaziachmetovas $^{\mathrm{b}}$, A. Salazar ${ }^{\mathrm{c}}$ \\ ${ }^{a}$ Department of communications Engineering, Universidad Miguel Hernández de Elche, Avda. Universidad \\ S/N, Edif. Quorum V, 03203, Elche (Alicante), Spain \\ Tel: (34) 966658 825 - Fax: (34) 966658903 - email: arodriguezm@goumh.es \\ ${ }^{b}$ Department of Signal Processing, Kaunas University of Technology, Studentu St. 48, LT-51367, Kaunas, \\ Lithuania \\ ${ }^{c}$ Department of Communications, Universidad Politécnica de Valencia, Avda. Naranjos S/N, 46022, \\ Valencia, Spain
}

\begin{abstract}
A new method for the automatic and simultaneous measurement of phase velocity and thickness for thin composite plates was developed based on Ping He's method, without any need of a priori knowledge of the material parameters. Two composites were analyzed: a block of clean epoxy and a thin specimen of glass-fiber reinforced plastic produced by Resin Transfer Molding. The proposed method combines cross-correlation functions and iterative deconvolution for accurate measurement of times of flight and gating. The new method has demonstrated to be more accurate than conventional Ping He's method, and can be implemented automatically thus saving processing time and increasing accuracy.

Keywords: Composite characterization, phase velocity, time of flight estimation, iterative deconvolution, thickness estimation
\end{abstract}

\section{Introduction}

The number of new composite materials has greatly increased in recent years, given to its low cost, ease of manufacture and the large number of applications they have. As these are experimental materials, it is essential to have the tools to control and evaluate the manufacturing process and the behavior of their characteristics with time and use. Ultrasound atten-

\footnotetext{
${ }^{*}$ Corresponding author

Email addresses: arodriguezm@goumh.es (A. Rodríguez), linas.svilainis@ktu.lt (L. Svilainis), vytautas.dumbrava@ktu.It (V. Dumbrava), andrius.chaziachmetovas@ktu.It (A. Chaziachmetovas), asalazar@dcom.upv.es (A. Salazar)
} 
uation and dispersion (changes in phase velocity with frequency) are two material properties directly related to the characteristics of the material and are widely used in non-destructive evaluation and material characterization.

Although it can be found a lot of techniques in literature for frequency dependent parameters measurement [1-9], Ping He's method [10-13] is one of the most used due to its ease and because it does not need any a priori knowledge of the material characteristics. Using simultaneous pulse-echo and through-transmission immersion measurements, it is able to provide the frequency dependent thickness and phase velocity. It is not an exact method and has several limitations $[10,13]$, but in spite of them, it still provides very useful results when the parameters of the material are unknown, which is especially interesting when analyzing fiber reinforced composites manufactured using RTM or sputtering technology, whose final inner composition is difficult to control due to its fabrication process [9, 14-16].

Unfortunately, Ping He's method has some drawbacks, especially those related to the procedure followed to avoid phase uncertainty, that affect accuracy and axial resolution, in addition that it needs manual fitting of some parameters, therefore it cannot be applied automatically. In this work we propose a modification of Ping He's method. New method is based on the cross-correlation processing and the iterative deconvolution. Thanks to such approach manual selection of the time shift can be avoided. Additionally, accuracy and axial resolution of the measurements are increased.

The proposed method is very accurate in the measurements if the media can be considered as low or moderate dispersive. Otherwise, cross-correlation is severely affected by dispersion of the pulses, thus accuracy in the gating procedure and thickness measurements will be compromised, and other solutions for high dispersive media should be considered, as proposed in $[17]$.

Section II briefly reviews Ping He's method and related problems. Section III is devoted to explain the proposed procedure, and section IV shows the experiment setup and the results of the analysis. Finally, section V summarizes the most relevant conclusions of this work. 


\section{A Review of Ping He's Method}

\subsection{Measurements of thickness and sound velocity}

The proper characterization of the dispersion and attenuation of the ultrasound in the frequency domain requires accurate measurements of the thickness of the specimen to be analyzed, which also implies a precise knowledge of the propagation speed in the material. Since our hypothesis is precisely the lack of any of these parameters, the first task is to obtain a simultaneous measurement of the speed of sound and thickness, for which we use the scheme of immersion measurements such as the one shown in Fig. 1 according to the procedure described in [13].

Figure 1: Experiment set-up of through-transmission and pulse-echo measurements.

The advantage of this method is that it only requires three measurements while keeping the layout of the transducers. First, the specimen (thickness $L$ and sound velocity $c_{s}$ ) is inserted between transducers at appropriate distances $\left(d_{1}\right.$ and $\left.d_{2}\right)$. Then, transducer $T_{1}$ sends a pulse and simultaneous reflected and passed through signals are recorded in transducer $T_{1}$ and $T_{2}$ respectively as $s_{T}(t)$ and $s_{R}(t)$. Note that $s_{R}(t)$ contains reflections from front and back surfaces from the specimen, which will be later gated and separated as $s_{R 1}(t)$ and $s_{R 2}(t)$ respectively. Finally, the specimen is removed and transducer $T_{1}$ sends a pulse through the water-path, which is received in transducer $T_{2}$ and recorded as $s_{W}(t)$. Although not strictly necessary, additional water-path measurement can be done changing the distance between transducers for an accurate measurement of the velocity in water.

Now, velocity and thickness can be measured $[1,4]$ using:

$$
c_{s}=c_{W} \cdot\left(\frac{2 \cdot\left(t_{W}-t_{T}\right)}{\left(t_{R 2}-t_{R 1}\right)}+1\right)
$$

and

$$
L=\frac{c_{W}}{2} \cdot\left(2 \cdot\left(t_{W}-t_{T}\right)+\left(t_{R 2}-t_{R 1}\right)\right),
$$

where $L$ and $c_{s}$ are the specimen thickness and speed of sound, $c_{W}$ is the speed of sound in water, and $t_{W}, t_{T}, t_{R 1}$ and $t_{R 2}$ are the times of flight of $s_{W}(t), s_{T}(t), s_{R 1}(t)$ and $s_{R 2}(t)$ respectively. 


\subsection{Measurements of frequency dependent parameters}

Let's call $A_{W}(f) e^{-\theta_{W}(f)}, A_{T}(f) e^{-\theta_{T}(f)}, A_{R 1}(f) e^{-\theta_{R 1}(f)}$ and $A_{R 2}(f) e^{-\theta_{R 2}(f)}$ the Fourier Transform of $s_{W}(t), s_{T}(t), s_{R 1}(t)$ and $s_{R 2}(t)$ respectively. Then, using [10,11, 13] and assuming negligible the dispersion in water [18], the phase velocity in the specimen $V_{P}(f)$ can be calculated as:

$$
V_{P}(f)=c_{w} \cdot\left[1+2 \cdot \frac{\theta_{W}(f)-\theta_{T}(f)}{\theta_{R 2}^{\prime}(f)-\theta_{R 1}(f)}\right],
$$

where $\theta_{W}(f), \theta_{T}(f), \theta_{R 1}(f)$ and $\theta_{R 2}^{\prime}(f)$ are the absolute phase spectra in the frequency domain of $s_{W}(t), s_{T}(t), s_{R 1}(t)$ and $-s_{R 2}(t)$ respectively (the negative sign of $s_{R 2}(t)$ is necessary to take into account the inherent $180^{\circ}$ inversion of the second echo due to reflection in back surface), $f$ is the frequency and $c_{W}$ is the speed of sound in water. Note that (3) does not depend on the speed of sound neither on the thickness of the specimen, so its calculation is not required.

Although not strictly necessary, we can calculate an estimate of the frequency dependent thickness using the phase velocity [11] as:

$$
L(f)=\frac{c_{W}}{4 \pi f} \cdot\left[2 \cdot\left(\theta_{W}(f)-\theta_{T}(f)\right)+\left(\theta_{R 2}^{\prime}(f)-\theta_{R 1}(f)\right)\right] .
$$

As stated in [11], the frequency dependence of the components of the right side in (4) should be canceled out resulting in the same constant thickness $L$ formulated in (2), but in practice it will change with frequency as it is calculated from experimental data. The mean of the calculated $L(f)$ within the frequency range of the measurement can be used as an estimate of the thickness $L$, and its standard deviation can be used to judge the reliability of the different methods [11].

It should be noted that absolute phases in (3) and (4) are affected by the $2 m \pi$ ambiguity due to the rapid phase change produced by the time delay [18]. Fig. 2 shows an example of the phase spectra of a signal having large offset from the origin (gray solid line).

Figure 2: Example of Ping He's unwrapping method.

Ping He developed a simple procedure in order to avoid the phase uncertainty due to phase unwrapping $[10,11,19]$. First, each pulse $s_{i}(t)$ is windowed and circularly shifted to the left until its centroid coincides with the origin of the window. Then, phase spectra of the 
shifted pulses are calculated using their respective Fourier Transforms, so that the relation between the phases of the original and shifted pulses are as follows:

$$
\theta_{i}(f)=\phi_{i}(f)+2 \pi f t_{i}^{\prime},
$$

where $\theta_{i}(f)$ is the absolute phase spectrum of $s_{i}(t), \phi_{i}(f)$ is the phase spectrum of the shifted version of $s_{i}(t)$, and $t_{i}^{\prime}$ is the time shift applied, which should include the difference between windows origin (trigger delay) if any. Additional time shifting can be applied to reduce any possible discontinuity in the selected frequency range, and should also be included in $t_{i}^{\prime}$.

Solid black line in Fig. 2 show the phase spectrum the same signal shifted left to its centroid position. It can be seen that shifting the signal to zero position reduces only the phase ramp, but variation in phase due to transducer and transmission function remains (note the dashed line for unwrapped case).

Figure 3: Example of Ping He's method operation. (a) Water-path A-scan in time domain and (b) its phase spectrum. (c) Passed-Through A-scan in time domain and (d) its phase spectrum. (e) Pulse-echo A-scan in time domain and (f) phase spectrum of first reflection (solid line) and second reflection (striped line) after shifting.

The procedure becomes more complicated when signal $s_{R}(t)$ is analyzed. First reflection $\left(s_{R 1}(t)\right)$ and second reflection $\left(s_{R 2}(t)\right)$ have to be gated and shifted to their corresponding origins. Gate position has to be selected manually, using a priori velocity and thickness data. Even after such operation problem indicated in Fig. 2 remains.

Figure 3 is used to demonstrate the method operation with signals obtained from a test specimen (glass fiber reinforced plastic). Solid lines in figures 3a, 3c and 3e show examples of water-path, passed-through and pulse-echo pulses respectively. Stripped lines in figures 3a and $3 \mathrm{c}$ show the shifted versions of the respective pulses using their corresponding centroids (named in the figure as $T_{C W}$ and $T_{C T}$ respectively), while stripped lines in Fig. 3e show the gating windows with the corresponding centroids (named in the figure as $T_{R 1}$ and $T_{R 2}$ respectively). Figures $3 \mathrm{~b}$ and $3 \mathrm{~d}$ show the phase spectra before (solid line) and after (striped line) shifting, where uncertainty has been removed. For the pulse-echo experiment, Fig. 3f shows the corresponding phase spectra of first (solid line) and second (striped line) reflections after shifting gated pulses to their centroid. 
Finally, once phase spectra are calculated, using (5) in (3) and (4), phase velocity $V_{P}(f)$ and thickness $L(f)$ can be calculated as:

$$
V_{P}(f)=c_{W} \cdot\left[1+2 \cdot \frac{\phi_{W}(f)-\phi_{T}(f)+2 \pi f\left(t_{W}^{\prime}-t_{T}^{\prime}\right)}{\phi_{R 2}^{\prime}(f)-\phi_{R 1}(f)+2 \pi f\left(t_{R 1}^{\prime}-t_{R 2}^{\prime}\right)}\right]
$$

and

$$
\begin{aligned}
L(f)=\frac{c_{W}}{4 \pi f} \cdot\left[2 \cdot\left(\phi_{W}(f)-\phi_{T}(f)\right)+\left(\phi_{R 2}^{\prime}(f)-\phi_{R 1}(f)\right)\right. \\
\left.+2 \pi f\left(t_{R 1}^{\prime}-t_{R 2}^{\prime}+t_{W}^{\prime}-t_{T}^{\prime}\right)\right]
\end{aligned}
$$

where $c_{W}$ is the sound velocity in water, $\phi_{W}(f), \phi_{T}(f), \phi_{R 1}(f)$ and $\phi_{R 2}(f)$ are the phase spectra of the respective shifted versions of $s_{W}(t), s_{T}(t), s_{R 1}(t)$ and $-s_{R 2}(t)$ (note again the extra $180^{\circ}$ phase shift applied to $\left.s_{R 2}(t)\right)$, and $t_{W}^{\prime}, t_{T}^{\prime}, t_{R 1}^{\prime}$ and $t_{R 2}^{\prime}$ are their respective time shift.

\subsection{Resume of Ping He's Method}

Concerning the methodology, Ping He's method has some problems related to the procedure followed to calculate the phases and the times of flight:

(i) transducers introduce the nonlinear phase response over frequency therefore simple phase shifting is ineffective;

(ii) selecting the centroid of the signal as reference point for time shifting does not lead to correct alignment of the phases, as the centroid provides a measure of the mean (it is calculated by integration of the energy) of the dispersion;

(iii) according to the original method time shift has to be applied in samples since time axis is discrete, though the actual shift could be a fraction of the samples;

(iv) even if subsample time shift is applied, additional manually selectable time shift have to be added to all signals to avoid the phase wrapping, which is especially complicated taking into account that such procedure has to be followed for four signals;

(v) as first and second reflections in reflected signal have to be gated, there is a compromise between the thickness and the axial resolution, which limits the minimum measurable 
thickness and/or the length of the excitation pulse used [10, 12]; furthermore, pulses are gated using rectangular windows manually for each measurement point, thus results will be compromised by the gating process (size and shape of the window directly affect the centroid position).

Of course, there are other sensitive points that affect the accuracy and precision of this method and that are common to this sort of techniques, as the phase cancellation at the receiving aperture [13], deviation from perfectly-normal incidence at both surfaces, the flatness of the gain spectrum [13], phase response of the receiving amplifier [10], diffraction loss [13], etc. We will only focus on the problems related to the methodology required to derive the measurements.

\section{New Procedure}

To overcome the aforementioned problems, we suggest using a combination of cross correlation and iterative deconvolution, as introduced in [20]. Correlation in frequency domain implies multiplication by the conjugate version of one of the spectra, thus the contribution in the phase due to all the sources of dispersion (transducers, water-path and transmission and/or reflection coefficients) are negated and only information of the specimen remains in the resulting phase difference. Cross-correlation acts also as a pulse compressor while retaining frequency information which will improve the gating process, besides it can be used for accurately estimate time of flight of the signals. Iterative deconvolution is used to disassemble the signal into separate reflections, thus providing the tool for automated time of flight values extraction which can be used for pulse gating.

We will start discussing the procedure designed for time of flight estimation, as it is crucial for the accuracy of the whole system.

\subsection{Time of flight estimation}

In the general time of flight estimation problem, and assuming the material as low or moderate dispersive, the received signal $s_{R}(t)$ can be considered as a delayed and attenuated version of the transmitted signal $s_{T}(t)$ embedded in noise:

$$
s_{R}(t)=a(t) \cdot s_{T}(t-T O F)+n(t),
$$


where $a(t)$ is the attenuation function and $n(t)$ is an uncorrelated additive white Gaussian noise. The goal of the time of flight (TOF) measurement is to estimate the true delay of signal arrival once it has propagated through the material. In simple applications, TOF is computed using the thresholding technique [21,22]. This technique offers a low cost and a simple solution, but has a poor accuracy even if high sample rate [23] is used since it does not explore all energy of the signal. Using several zero crossings or application of some integral estimate like center of area (centroid) [24] allow for results improvement, yet lacks the matched filter property to align the frequency components phases.

The direct correlation technique uses the peak position of the cross-correlation function (or matched filter output) [25] between the received and the transmitted signals. As the signal and the noise are orthogonal, cross-correlation $x_{R T}(t)$ between transmitted and received signals will lead to:

$$
\begin{aligned}
x_{R T}(\tau) & =E\left\{s_{R}(t) s_{T}(t-\tau)\right\} \\
& =E\left\{\left(a(t) \cdot s_{T}(t-T O F)+n(t)\right) \cdot s_{T}(t-\tau)\right\} \\
& =a(t) \cdot E\left\{s_{T}(t-T O F) s_{T}(t-\tau)\right\} \cdot E\left\{n(t) \cdot s_{T}(t-\tau)\right\} \\
& =a(t) \cdot x_{T T}(\tau-T O F),
\end{aligned}
$$

where $x_{T T}(\tau)$ is the auto-correlation function of the transmitted signal. Thus, in this context, the peak position of the cross-correlation function will provide the best estimate of the TOF $[26]$.

In discrete time domain, the peak of the correlation function is found by interpolating the discrete version of the cross-correlation function $x_{R T}[m]$ (Fig. 4 insert):

$$
x_{R T}[m]=\sum_{k=1}^{K} s_{R}[k] s_{T}[m-k],
$$

where $s_{R}[k]$ and $s_{T}[m-k]$ are the discrete versions of transmitted and received signals at discrete time instants $k$.

Figure 4: Example of time of flight estimation in discrete time domain. Insert: Detail around the peak of the cross-correlation.

In ideal case sinc function should be used for the TOF estimation between samples, but it is not possible in real time application. Although several truncated interpolation techniques 
exist [25, 27], cosine fitting techniques have recently demonstrated to produce the smallest bias error [26]. According to [26, 27], the estimated subsample shift that should be added is given by the following expression:

$$
\Delta T O F=-\frac{\theta}{F_{S} \omega_{0}}
$$

with

$$
\begin{gathered}
\omega_{0}=\arccos \left(\frac{x_{R T}[M-1]+x_{R T}[M+1]}{x_{R T}[M]}\right), \\
\theta=\arctan \left(\frac{x_{R T}[M-1]-x_{R T}[M+1]}{2 x_{R T}[M] \sin \omega_{0}}\right)
\end{gathered}
$$

where $F_{S}$ is the sampling frequency and $M$ the discrete peak position of $x_{R T}[m]$ (Fig. 4 insert), thus the final subsample TOF estimate will be:

$$
T O F=\frac{M}{F_{S}}+\Delta T O F,
$$

This procedure can achieve a resolution in the order of picoseconds [26], while maximum resolution achieved using centroid is in the order of nanoseconds [27], thus an improvement in the thickness and phase velocity measurements is expected. Furthermore, due to matched filter properties to align the frequency components of the phases, cross-correlation method is less sensitive to dispersion than centroid based methods [24, 26].

As we will see in next sections, applying the previous formulation to our experiment and considering the through-transmitted signal as the received signal and the water-path signal as the transmitted signal, TOF between them is obtained. Calculations can be repeated using first and second echo in pulse-echo experiment as transmitted and received signals respectively, thus obtaining the TOF between them.

\subsection{Through-Transmission parameters}

For the through-transmission experiment, cross-correlation $x_{T W}(t)$ between the passedthrough signal and the water-path signal can be calculated in frequency domain using their respective Fourier Transform (FT) as:

$$
\begin{gathered}
x_{T W}(t)=s_{T}(t) \circ s_{W}(t) \stackrel{\mathrm{FT}}{\longrightarrow} X_{T W}(f)=\left(S_{T}(f)\right)^{*} \cdot S_{W}(f) \\
X_{T W}(f)=A_{T}(f) \cdot A_{W}(f) \cdot e^{-j\left(\theta_{W}(f)-\theta_{T}(f)\right)}=A_{T W}(f) \cdot e^{-j \theta_{T W}(f)}
\end{gathered}
$$


where $A_{T W}(f)$ is the amplitude spectrum of $X_{T W}(f)$ and $\theta_{T W}(f)$ is its phase spectrum, which is actually the desired difference between the absolute phases of the signals in (3), where only information of the specimen remains as the rest of the phase components cancel each other. This gives an additional advantage: different transducers can be used for transmission and reception.

Now $x_{T W}(t)$ is calculated as the inverse FT of $X_{T W}(f)$. As mentioned in the previous section, the delay of the peak of the resulting cross-correlation is actually the time of flight between pulses $\left(T O F_{T W}\right)$. Note also that it can be used in (1) and (2) to obtain the speed of sound and thickness.

Now, the cross-correlation result is shifted using the $T O F_{T W}$ calculated previously in order to avoid the $2 m \pi$ ambiguity in the phase. To take advantage of the accuracy achieved in $T O F_{T W}$ calculation, time shift is performed in a subsample basis using the frequency domain $[26,28,29]$, so that the shifted version of $x_{T W}(t)$ is calculated as:

$$
x_{T W}\left(t-T O F_{T W}\right)=I F T\left(F T\left(x_{T W}(t)\right) \cdot e^{-j \omega T O F_{T W}}\right) .
$$

Then, phase difference $\phi_{T W}(f)$ can be calculated as the phase of the Fourier transform of $x_{T W}\left(t-T O F_{T W}\right):$

$$
\phi_{T W}(f)=\arctan \left\{F T\left(\left(t-T O F_{T W}\right)\right)\right\} .
$$

Finally, the desired absolute phase difference $\theta_{T W}(f)$ is obtained using (5) as:

$$
\theta_{T W}(f)=\theta_{W}(f)-\theta_{T}(f)=\phi_{T W}(f)+2 \pi f \cdot T O F_{T W}
$$

Figure 5 is used to demonstrate the method operation. Solid line in Fig. 5a shows the result in time domain of the cross correlation between passed-through and water-path pulses in figures $3 \mathrm{a}$ and $3 \mathrm{~b}$ respectively, while stripped line shows its shifted version using the peak, i.e. $T O F_{T W}$. Fig. $5 \mathrm{~b}$ shows the phase of this shifted version (stripped line) that can be compared with the phase difference obtained with Ping He's method (solid line), where it can be seen the improvement in the phase alignment achieved with the new method, which produces almost straight line of phase response.

Figure 5: Example for through-transmission parameters calculation using cross-correlation. (a) Cross-correlation between water-path and through-transmission signals in time domain. 
(b) Comparison of resulting phase differences using Ping He's method (solid line) and new method (gray stripped line).

\subsection{Pulse-echo parameters}

A similar procedure can be followed to obtain the phase difference between the reflected signals. If the system is considered as linear the received signal in pulse-echo experiment can be written as the contribution of the successive echoes:

$$
s_{R}(t)=s_{R 1}(t)+s_{R 2}(t)+\sum_{j>2} s_{R j}(t)
$$

where $s_{R 1}(t)$ and $s_{R 2}(t)$ are the first and second reflection respectively, and $s_{R j}(t)$ the successive reflections which are not needed and thus gated. Then, cross-correlation between $s_{R}(t)$ and water-path signal $s_{W}(t)$ is calculated as:

$$
\begin{aligned}
x_{R W}(t) & =s_{R}(t) \circ s_{W}(t)=s_{R 1}(t) \circ s_{W}(t)+s_{R 2}(t) \circ s_{W}(t) \\
& =x_{R 1 W}(t)+x_{R 2 W}(t)
\end{aligned}
$$

where $x_{R 1 W}(t)$ and $x_{R 2 W}(t)$ are the respective cross-correlations between the echoes and the water-path signal, whose Fourier Transform can be written respectively as:

$$
X_{R 1 W}(f)=A_{R 1}(f) \cdot A_{W}(f) \cdot e^{-j\left(\theta_{W}(f)-\theta_{R 1}(f)\right)}
$$

and

$$
X_{R 2 W}(f)=A_{R 2}(f) \cdot A_{W}(f) \cdot e^{-j\left(\theta_{W}(f)-\theta_{R 2}(f)\right)} .
$$

Note that due to cross-correlation properties [30-34], $x_{R 1 W}(t)$ and $x_{R 2 W}(t)$ are compressed versions of the original echoes $s_{R 1}(t)$ and $s_{R 2}(t)$ that retain the original frequency information thus its separation will be easier. Once these are properly gated, cross-correlation $x_{R 12 W}(t)$ between them can be calculated in frequency domain as:

$$
\begin{aligned}
X_{R 12 W}(f) & =\left(X_{R 1 W}(f)\right)^{*} \cdot X_{R 2 W}(f) \\
& =A_{R 1}(f) \cdot A_{R 2}(f) \cdot A_{W}^{2}(f) \cdot e^{-j\left(\theta_{R 2}(f)-\theta_{R 1}(f)\right)},
\end{aligned}
$$

whose phase is precisely the difference between the absolute phases of the first and second reflection $\theta_{R}(f)=\theta_{R 2}(f)-\theta_{R 1}(f)$ in (4), where only information of the specimen remains. 
As we made with through-transmission signal, if $x_{R 12 W}(t)$ is calculated as the inverse FT of $X_{R 12 W}(f)$, the delay of the peak of the resulting signal is actually the time of flight between reflections $\left(T O F_{R}\right)$, that can be used for the time shift needed to remove the phase uncertainty of $\theta_{R}(f)$ and to solve (1) and (2) to obtain the speed of sound and thickness.

Challenge now is how to perform the gating of $x_{R 1 W}(t)$ and $x_{R 2 W}(t)$ automatically, for which we will use the iterative deconvolution.

\subsection{Iterative deconvolution}

Iterative deconvolution $[28,35-40]$ assumes that if received signal is the sum of the step responses at each reflector, reflections can be separated subtracting a reference signal properly placed and weighted. In our case, iterative deconvolution is performed as follows:

(i) Calculate cross-correlation (Fig. 6a) between the input signal $s_{R}(t)$ and the water-path signal $s_{W}(t)$ used as reference. The maximum will be the first peak and will coincide with the time of flight, $T O F_{R 1}$, between first echo $s_{R 1}(t)$ and reference $s_{W}(t)$.

$$
x_{R W}(t)=s_{R}(t) \circ s_{W}(t) \stackrel{\text { peak }}{\longrightarrow} T O F_{1}
$$

(ii) Shift the reference by $T O F_{1}$ in a subsample basis in frequency domain, weight it and subtract it to the input to obtain the remainder $r(t)$ (Fig. 6b).

$$
\begin{aligned}
& s_{W}^{\prime}(t)=s_{W}\left(t-T O F_{1}\right) \\
& A=\frac{\frac{1}{N} \sum_{k=1}^{N} s_{W}^{\prime}[n] \cdot s_{R} n}{\frac{1}{N} \sum_{k=1}^{N}\left(s_{W}^{\prime}[n]\right)^{2}} \\
& r(t)=s_{R}(t)-A \cdot s_{W}^{\prime}(t)
\end{aligned}
$$

(iii) Repeat step 1 with the remainder of the previous subtraction (Fig. 6c) to obtain the time of flight $T O F_{2}$ between reference $s_{W}(t)$ and second echo $s_{R 2}(t)$. Remainder is negated before correlation to take into account the inversion caused by reflection in back surface.

$$
x_{r W}(t)=-r(t) \circ s_{W}(t) \stackrel{\text { peak }}{\longrightarrow} T O F_{2}
$$

Figure 6: Example iterative deconvolution operation. (a) Cross-Correlation between water-path (Fig. 3a) and pulse-echo (Fig. 3c) signals. (b) Remainder after first iteration. (c) 
Cross-correlation between remainder and water-path. (d) Cross-correlation and gating windows. (e) Cross-correlation between gated cross-correlations. (f) Resulting phase spectrum using Ping He's (solid line) and the new method (stripped line).

There are several choices for the reference signal [28, 36, 40-42]: the mean of all the first reflections after aligning them to a reference point, a reflection from a polished and flat reflecting surface of the same material, a reflection from an ideal reflector (usually aluminum) and finally just the water-path signal from the through-transmission experiment. We have chosen this latter because it does not need any additional calculation neither new signal acquisition. According to our test, although not totally removed, reduction of first echo achieved with (26) using water-path signal as reference is enough to make prevailing the peak of second cross-correlation.

Note that in this example first echo is ahead in time than water-path signal (TOF 1 is positive), while second echo is delayed respect to water-path ( $T O F_{2}$ is negative). Note also that difference between them, $T O F_{R}=T O F_{2}-T O F_{1}$, is the time of flight between crosscorrelations, and as they have been calculated with the same reference, it is actually the time of flight between echoes $s_{R 2}(t)$ and $s_{R 1}(t)$ and can be used directly in (1) and (2).

Cross-correlations are now gated in time domain using an optimized Tukey window $g(t)$ [43]. If we assume that $x_{R W}(t)$ in (22) is calculated in frequency domain, then $s_{R 1 W}(t)$ and $s_{R 2 W}(t)$ can be gated as:

$$
\begin{aligned}
& x_{R 1 W}(t)=x_{R W}\left(t-T O F_{1}\right) \cdot g(t) \\
& x_{R 2 W}(t)=x_{R W}\left(t-T O F_{2}\right) \cdot g(t) .
\end{aligned}
$$

Figure 6d shows the gating process and Fig. 6e shows the cross-correlation between gated signals, whose phase is precisely the desired absolute phase difference between echoes (21).Now, $x_{R 12 W}(t)$ is circularly shifted (striped line in Fig. 6e) in a subsample basis until its peak (which is actually the time of flight $T O F_{R}$ ) coincides with the origin (shift to the right, as second echo will always be delayed respect to first echo, just the opposite that between passed-through and water-path). Striped line in Fig. 6 f shows the phase difference $\phi(f)=\phi_{2 W}(f)-\phi_{1 W}(f)$ calculated as the phase of the Fourier transform of the delayed version of $x_{R 12 W}(t)$, where the desired phase alignment without ambiguity can be easily seen, 
specially if compared with results obtained using Ping He's method (solid line).

Now, if we calculate the phase difference as:

$$
\phi_{R}(f)=\arctan \left\{F T\left(\left(t-T O F_{R}\right)\right)\right\}
$$

the desired absolute phase difference $\theta_{R}(f)$ is obtained using (5) as:

$$
\theta_{R}(f)=\theta_{R 2 W}(f)-\theta_{R 1 W}(f)=\phi_{R}(f)+2 \pi f \cdot T O F_{R}
$$

Finally, using the notation followed so far, equations (1), (2), (6) and (7) can now be written respectively as:

$$
\begin{gathered}
c_{s}=c_{W} \cdot\left(\frac{2 \cdot\left(T O F_{T W}\right)}{\left(T O F_{R}\right)}+1\right), \\
L=\frac{c_{W}}{2} \cdot\left(2 \cdot\left(T O F_{T W}\right)+\left(T O F_{R}\right)\right), \\
V_{P}(f)=c_{w} \cdot\left[1+2 \cdot \frac{\phi_{T W}(f)+2 \pi f \cdot T O F_{T W}}{\phi_{R}(f)+2 \pi f \cdot T O F_{R}}\right],
\end{gathered}
$$

and

$$
L(f)=\frac{c_{W}}{4 \pi f} \cdot\left[2 \cdot \phi_{T W}(f)+\phi_{R}(f)+2 \pi f\left(2 \cdot T O F_{T W}+T O F_{R}\right)\right] .
$$

\section{Experiments and results}

\subsection{Experiment setup}

A typical setup for immersion experiment was used to develop the measurements according to Fig. 1. Transmitter $T_{1}$ was a $5 \mathrm{MHz}$ wideband focused transducer IRY405 from NDT Transducers LLC and receiver T2 was a composite $5 \mathrm{MHz}$ transducer TF5C6 from Doppler Electronic Technologies. Such pair of transducers gives a frequency range of $0.5-7 \mathrm{MHz}$ at $-20 \mathrm{~dB}$. The distance between transducer $T_{1}$ and the specimen was set to the focal distance (36 $\mathrm{mm}$ in this case), and $T_{2}$ was placed at $10 \mathrm{~mm}$ from back surface. The pulser-receiver used was SE-TX06-00, with sampling frequency of the acquisition system set to $100 \mathrm{MHz}$ and sampling windows length adjusted in order to have all measurements of each experiment in the same time basis. A 100 ns $5 \mathrm{MHz}$ pulse has been used as excitation.

Two specimens where analyzed (Fig. 7): A test piece of clear clean epoxy (CCE) of constant thickness $(6.9 \pm 0.05 \mathrm{~mm}$, caliper measurement) and a piece of fiber glass reinforced 
plastic (FGRP) produced by resin transfer molding with uneven faces and variable thickness (1.8-2.1 $\pm 0.05 \mathrm{~mm}$, caliper measurement). Epoxy specimen is used as control experiment, since it is assumed to be homogeneous and therefore much less dispersive than the composite. For both samples, $30 \mathrm{~A}$-scans were acquired at different locations in steps of $0.5 \mathrm{~mm}$ moving the specimens along $\mathrm{X}$ axis with an automated XY scanner. At each particular position 25 A-scans were acquired and averaged to improve the signal-to-noise ratio (SNR). Examples of the resulting A-scans acquired at a single location for the FGRP and CCE specimens can be seen in Fig. 8.

Figure 7: Analyzed specimens: CCE front and section views (left). FGRP front and section views.

For each experiment, sound velocity in water was calculated using two measurements with different distances between $T_{1}$ and $T_{2}$. Measurements were repeated systematic and automatically at different stages of the process, and obtained values were averaged providing an accurate measurement for each experiment independent of temperature.

\subsection{Results}

Figure 8 show examples of A-scans acquired at a particular position for the CCE (left) and FGRP (right) specimens respectively. From up to down, it shows the passed-through and pulse-echo signals respectively. Second echo in CCE specimen has been magnified (x15) in Fig. 8c only for visualization purposes, as it was severely attenuated.

Figure 8: Example of A-scans. (a) CCE passed-through. (b) FGRP passed-through. (c) CCE pulse-echo. (c) FGRP pulse-echo.

For the CCE specimen (control specimen), it can be clearly seen (Fig. 8c) that axial resolution is not compromised. Concerning the FGRP specimen, resonances due to the thickness of the specimen can be appreciated in passed-through signal (Fig. 8b around 2.2 $\mu s)$ and in the pulse-echo signal (Fig. $8 \mathrm{~d}$ around $4 \mu s$ ).

Results obtained using (1) and (2) for the thickness and speed of sound at each location are shown in Fig. 9 for both specimens and will be used as reference for further comparisons. Velocity measurements are consistent with nominal values: velocity in CCE is constant around nominal value (Fig. 9a) and it changes with position for FGRP (Fig. 9c) as it depends on the number, size and orientation of the fibers. In the other hand, calculated thickness agrees with 
caliper measurements, following the flatness of the CCE specimen (Fig. 9b) and the unevenness of the FGRP specimen (Fig. 9d). Note that caliper tolerance (red arrows) around caliper measure (red points) is 50 microns while precision in thickness using cosine interpolation and cross-correlation for times of flight estimation is in the order of nanometers.

Figure 9: Speed of sound and thickness.(a) Speed of sound for CCE (b) Thickness for CCE (c) Speed of sound for FGRP (d) Thickness for FGRP.

Next figures show a comparison of the results obtained using Ping He's method (red) and the proposed procedure (black) for the frequency dependent parameters. In all cases, first figure will depict the evolution of the variable (phase velocity or thickness) with frequency for all the scanning locations, resulting in a cloud of points at each frequency. Second figure will depict the difference between the frequency dependent variable and the absolute variable at each location and for each frequency. Finally, third figure will show the standard deviation of the previous error as a function of the frequency.

Figure 10 shows velocity results for the CCE specimen in all the scanning points. For both procedures results are very similar: velocity decays slowly with frequency as expected (Fig. 10a), and error is more or less the same (Fig. 10c). This is because the specimen thickness $(6.9 \mathrm{~mm})$ is much higher than the axial resolution $(\approx 0.52 \mathrm{~mm}$ considering pulseecho experiment for a $5 \mathrm{MHz} 100 \mathrm{~ns}$ pulse at $\approx 2600 \mathrm{~m} / \mathrm{s}$ ), so both procedures are able to separate the echoes properly. Standard deviation is slightly lower for the new procedure (Fig. 10e), due to the higher accuracy in the time of flight estimation, although in both cases it is very low due to the homogeneous nature of the specimen.

Figure 10: Comparison of phase velocity and thickness for the CCE specimen obtained using Ping He's method (red) and the new procedure (black). (a) Phase velocity in m/s (c) Bias in m/s (e) Standard deviation in m/s. (b) Thickness in mm (d) Bias in $\mu m$ (f) Standard deviation in $\mu m$.

Difference in accuracy is more evident if we analyze the thickness. Although behaving as expected (thickness constant with frequency) for both methods (Fig. 10b), error is clearly lower for the new procedure (Fig. 10c). Note that Ping He's shows a persistent off-set of around 100 microns. Accuracy in the time of flight calculation is the great advantage of the new procedure for low dispersive materials or where axial resolution is not compromised. 
Finally, although very low in both cases, standard deviation is also lower for the new method (Fig. 10f).

Concerning the FGRE specimen, Fig. 11 clearly reveal the effects of the loss of axial resolution and the presence of the fibers. Oscillations in lower frequencies show resonances due to specimen thickness (around 1.3 MHz the wavelength is comparable to the specimen thickness), while medium and high frequencies are affected (dispersed) by the size, number and orientation of the fibers. This is especially true for Ping He's method, for which the usable highest frequency has decreased to $5 \mathrm{MHz}$, while the new procedure still extends up to $6.5 \mathrm{MHz}$, as can be seen especially in the standard deviation (Fig. 11e and Fig. 11f). Accuracy is also affected in Ping He's method, as can be clearly appreciated in Fig. 11c and specially Fig. 11d where the off-set is about 400 microns.

It can also be seen that the standard deviation values (Fig. 11e and Fig. 11f) are much higher compared to those obtained for the CCE specimen (Fig. 10e and Fig. 10f). This is because the mentioned dispersion due to the heterogeneous distribution of the glass fibers at each scanning location.

Figure 11: Comparison of phase velocity and thickness for the FGRP specimen obtained using Ping He's method (red) and the new procedure (black). (a) Phase velocity in m/s (c) Bias in m/s (e) Standard deviation in m/s. (b) Thickness in mm (d) Bias in $\mu m$ (f) Standard deviation in $\mu m$.

This example clearly show the enhancement in the axial resolution and usable bandwidth achieved with the new procedure. Due to dispersion and loss of axial resolution pulses are overlapped, thus, after gating, resulting centroids used in Ping He's method are misadjusted, in addition that calculation of the correct time of flight is compromised.

On the other hand, compression achieved with cross-correlation makes that less frequencies are affected by overlap/gating, thus increasing the frequency range compared to Ping He's method. Properties of cross-correlation are not affected by dispersion, thus the peak of cross-correlation is still the best choice for phase flattening. Furthermore, it can be used for iterative deconvolution, thus if pulses are not severely overlapped, accuracy in the time of flight calculation is not compromised. 


\section{Conclusion}

In this work we have presented a new method for automatic and simultaneous measurement of the phase velocity and thickness for low or moderate composite materials based on Ping He's method and using cross-correlations and iterative deconvolution. Using cross correlations only two phase difference have to be calculated, instead of the four phases that needs the original method, thus reducing the sources of error.

Cross-correlation has demonstrated to be a powerful tool that improves the alignment of the frequency content of the phases thus improving the phase ambiguity removal procedure. It also provides an accurate measure of the time of flight, in addition that pulse compression achieved with correlation ease the gating of the reflected pulses, thus increasing the axial resolution and/or allowing the use of longer pulses.

On the other hand, all the process can be developed automatically combining crosscorrelation and iterative deconvolution, which has demonstrated to be very efficient in the measurement of the times of flight. Accuracy increases considerably if cosine interpolation and subsample shifting in frequency domain are used.

\section{Acknowledgment}

This research was funded by a project IN-SMART, grant No. VP1-3.1SMM-10-V-02-012 and by the Spanish Ministerio de Ciencia e Innovacion (TEC2011-23403).

[1] Hsu DK, Hughes MS. Simultaneous ultrasonic velocity and sample thickness measurement and application in composites. J Acoust Soc Am 1992;92(2):669-75.

[2] Graciet C, Hosten B. Simultaneous measurement of speed, attenuation, thickness and density with reflected ultrasonic waves in plates. Proc IEEE Ultrason Symp 2008 $1994 ;: 1219-$.

[3] Wang H, Juang W, Cao W. Characterization of lead zirconate titanate piezoceramic using high frequency ultrasonic spectroscopy. J Appl Phys 1999;85(12):8083-91.

[4] Fei D, Hsu DK, Warchol M. Simultaneous velocity, thickness and profile imaging by ultrasonic scan. Journal of Nondestructive Evaluation 2001;20(3):95-112. 
[5] Vangi D. Stress evaluation by pulse-echo ultrasonic longitudinal wave. Experimental Mechanics 2001;41(3):277-81.

[6] Higuti RT, Adamowski JC. Ultrasonic densitometer using a multiple reflection technique. IEEE Trans on Sonics and Ultras 2002;49(9):1260-8.

[7] Greco de sousa AV, Alburquerque Pereira WC, Machado JC. An ultrasonic theoretical and experimental approach to determine thickness and wave speed in layered media. IEEE Trans Ultrason Ferroelectr Freq Control 2007;54(2):386-93.

[8] Gómez Álvarez-Arenas ET. Simultaneous determination of the ultrasound velocity and the thickness of solid plates from the analysis of thickness resonances using air-coupled ultrasound. Ultrasonics 2010;50:104-9.

[9] Sun E, Cao W, Han P. Frequency dispersion of longitudinal ultrasonic velocity and attenuation in [001]c-poled 0.24pb(in1/2nb1/2)o30.45pb(mg1/3nb2/3)o30.31pbtio3 single crystal. IEEE Trans Ultrason Ferroelectr Freq Control 2011;58(8):1669-73.

[10] He P. Experimental verification of models for determining dispersion from attenuation. IEEE Trans Ultrason Ferroelectr Freq Control 1999;46(3):706-14.

[11] He P. Measurement of acoustic dispersion using both transmitted and reflected pulses. J Acoust Soc Am 2000;107(2):801-7.

[12] He P. Simultaneous measurement of sound velocity and wall thickness of a tube. Ultrasonics $2001 ; 39: 407-11$.

[13] He P, Zheng J. Acoustic dispersion and attenuation measurement using both transmitted and reflected pulses. Ultrasonics 2001;39:27-32.

[14] Wrobel G, Pawlak S. The effect of fiber content on the ultrasonic wave velocity in glass/polyester composites. J Achievements in Materials and Manufacturing Engineering 2007;20(1-2):295-8. 
[15] Adamowski JC, A. AM, Perez N, Buiochi F. A large aperture ultrasonic receiver for through-transmission determination of elastic constants of composite materials. Proc IEEE Ultrason Symp 2008 2008;:1524-7.

[16] Zhang C, Le HL, Zheng R, Ta D, Lou E. Measurements of ultrasonic phase velocities and attenuation of slow waves in cellular aluminum foams as cancellous bone-mimicking phantoms. J Acoust Soc Am 2011;129(5):3317-26.

[17] Perelli A, De Marchi L, Marzani A, Speciale N. Acoustic emission localization in plates with dispersion and reverberations using sparse pzt sensors in passive mode. Smart Mater and Struct 2012;21(2):025010.

[18] Wu J. Determining velocity and attenuation using broadband pulse technique. Acoustical Imaging 1997;23:137-43.

[19] Rokhlin SI, Lewis DK, Graff KF, Adler L. Realtime study of frequency dependence of attenuation and velocity of ultrasonic waves during the curing reaction of epoxy resin. J Acoust Soc Am 1986;79(6):1786-93.

[20] Svilainis L, Rodríguez A, Dumbrava V, Chaziachmetovas A, Eidukynas V. Estimation of composite plate attenuation. Electronics and Electrical Eng 2013;20(1):59-62.

[21] Ivchenko V, Kalashnikov AN, Challis RE, Hayes-Gill BR. High-speed digitizing of repetitive waveforms using accurate interleaved sampling. IEEE Trans Instrumentation and Measurement 2007;56(4):1322-8.

[22] Draudviliene L, Mazeika L. Analysis of the zero-crossing technique in relation to measurements of phase velocities of the s0 mode of lamb waves. Ultragarsas 2010;65(3):11-4.

[23] Afaneh A, Kalashnikov AN. Embedded processing of acquired ultrasonic waveforms for online monitoring of fast chemical reactions in aqueous solutions. Proc IEEE 6th IDAACS Conference 2011;::965-70.

[24] Kalashnikov AN, Shafran KL, Challis RE, Perry CC, Unwin ME, Holmes AK, et al. Super-resolution in situ ultrasonic monitoring of chemical reactions. Proc IEEE Ultrason Symp 1996 2004;:549-52. 
[25] Viola F, Walker WF. A spline-based algorithm for continuous time-delay estimation using sampled data. IEEE Trans Ultrason Ferroelectr Freq Control 2005;52(1):80-93.

[26] Svilainis L, Lukoseviciute K, Dumbrava V, Chaziachmetovas A. Subsample interpolation bias error in time of flight estimation by direct correlation in digital domain. Measurements 2013;46:3950-8.

[27] Céspedes I.and Huang Y, Ophir J, Spratt S. Methods for estimation of subsample time delays of digitized echo signals. Ultrasonic Imaging 1995;17:142-71.

[28] Svilainis L, Dumbrava V, Aleksandrovas A, Chaziachmetovas A, Kitov S, Pagodinas D. Signal stripping technique for ultrasonic imaging. Proc 5th Int Conf on NDT of HSNT-IC MINDT 2013 2013;:1-8.

[29] Svilainis L, Aleksandrovas A, Lukoseviciute K, Eidukynas V. Investigation of the time of flight estimation errors induced by neighboring signals. Proc IEEE 7th IDAACS Conference 2013;:413-8.

[30] El Guerjouma R, Mouchtachi A, Jayet Y, Baboux JC. Non destructive evaluation of graphite by ultrasonic velocity measurement using cross-correlation and hilbert transform methods. Proc IEEE Ultrason Symp 1992 1992;:829-32.

[31] Gan TH, Hutchins DA, Billson DR, Schindel DW. The use of broadband acoustic transducers and pulse-compression techniques for air-coupled ultrasonic imaging. Ultrasonics 2001;39:181-94.

[32] Pallav P, Hutchins DA, Yin X. Air-coupled ultrasonic spectroscopy of highly damping materials using pulse compression. IEEE Trans Ultrason Ferroelectr Freq Control 2009;56(6):1207-17.

[33] Svilainis L, Motiejunas G. Spread spectrum signal performance investigation in a bandlimited channel. Ultragarsas 2008;63(4):30-4.

[34] Svilainis L, Aleksandrovas A. Application of arbitrary pulse width and position trains for the correlation sidelobes reduction for narrowband transducers. Ultrasonics 2013;53:1344-8. 
[35] Barrodale I, Zala CA, Chapman NR. Comparison of the 11, and 12 norms applied to one-at-a-time spike extraction from seismic traces. Geophysics 1984;49:2048-52.

[36] Crilly PB. A quantitative evaluation of various iterative deconvolution algorithms. IEEE Trans Instrumentation and Measurement 1991;40(3):558-62.

[37] Anaya JJ, Ullate LG, Fritsch C. A method for real-time deconvolution. IEEE Trans Instrumentation and Measurement 1992;41(3):413-9.

[38] Xin J, Bilgutay NM. Ultrasonic range resolution enhancement using 11 norm deconvolution. Proc IEEE Ultrason Symp 1993 1993;:711-4.

[39] Kazys R, Svilainis L. Ultrasonic detection and characterization of delaminations in thin composite plates using signal processing techniques. Ultrasonics 1997;35:367-83.

[40] Li X, Li X, Liang W, Chen L. 10-norm regularized minimum entropy deconvolution for ultrasonic ndt \& e. NDT\&E Int 2012;47:80-7.

[41] Tanaka S, Jiang J, Takesue T. A model-based adaptive algorithm for determination of time-of-flight in ultrasonic measurement. Proc IEEE 36th SICE Conference 1997;:96570.

[42] Demirli R, Saniie J. Model-based estimation of ultrasonic echoes part ii: nondestructive evaluation applications. IEEE Trans Ultrason Ferroelectr Freq Control 2001;48(3):80311.

[43] Okarma K. Constructed polynomial windows with high attenuation of sidelobes. Electronics and Electrical Eng 2013;19(5):57-60. 


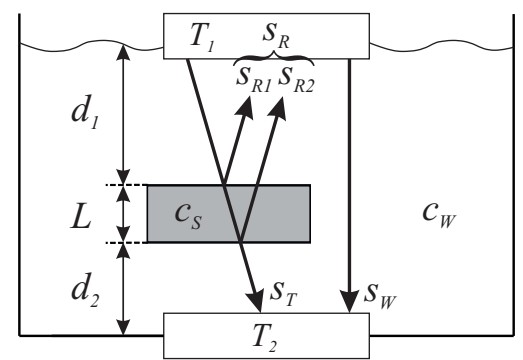

Figure 1: Experiment set-up of through-transmission and pulse-echo measurements.

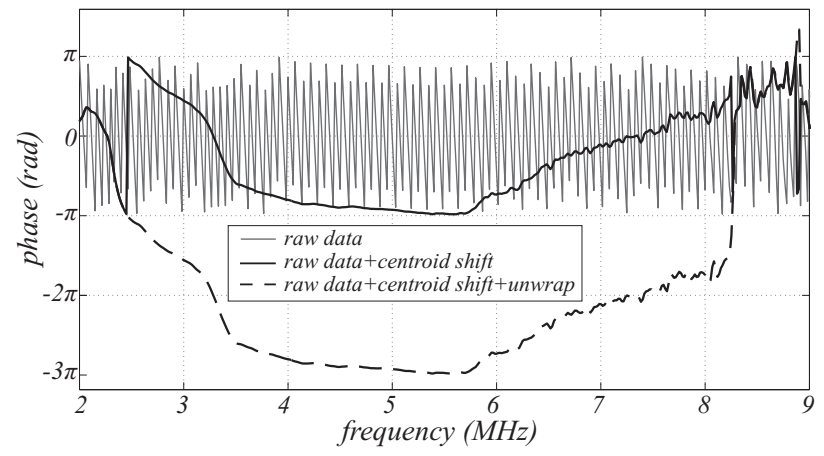

Figure 2: Example of Ping He's unwrapping method. 

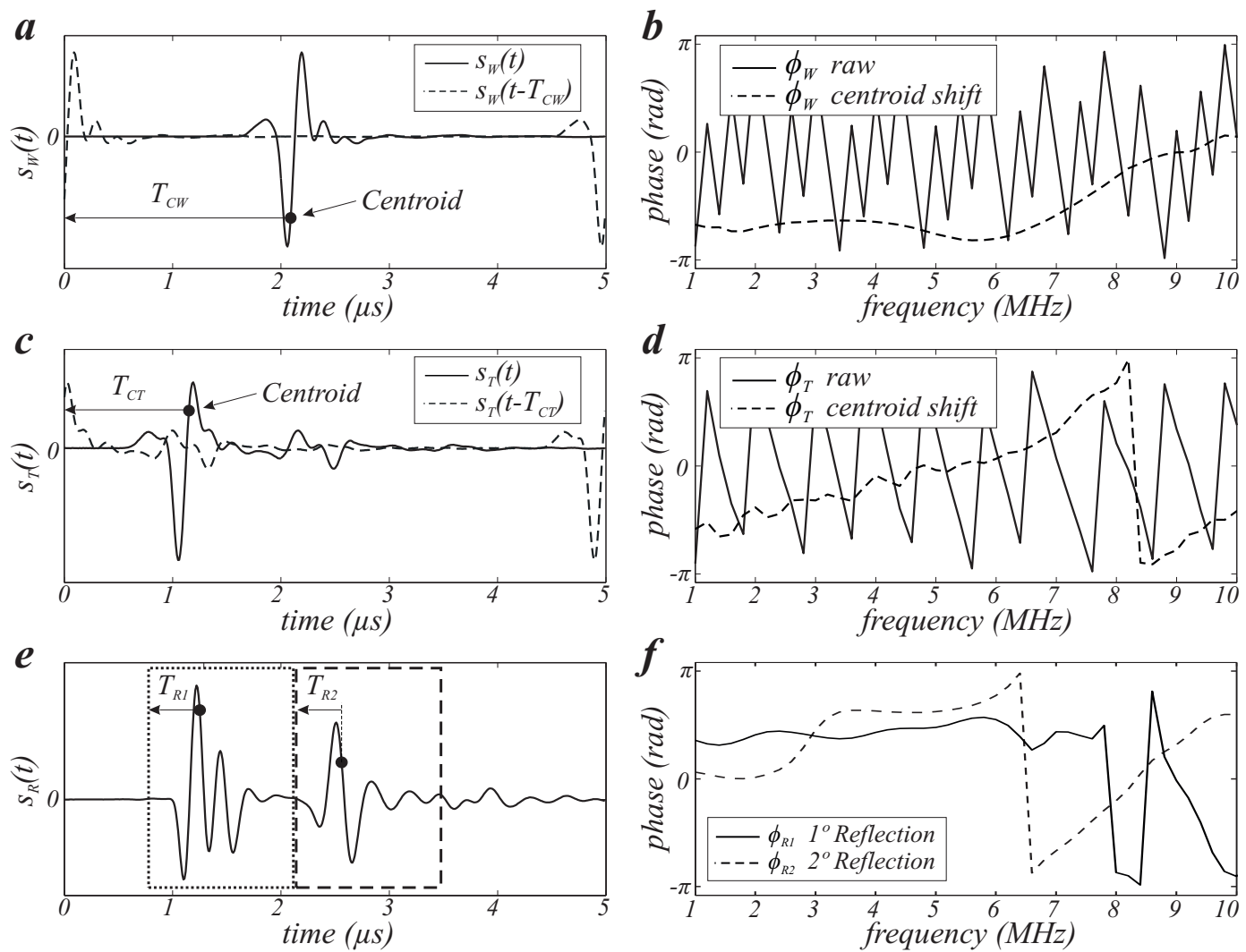

Figure 3: Example of Ping He's method operation. (a) Water-path A-scan in time domain and (b) its phase spectrum. (c) Passed-Through A-scan in time domain and (d) its phase spectrum. (e) Pulse-echo A-scan in time domain and (f) phase spectrum of first reflection (solid line) and second reflection (striped line) after shifting.

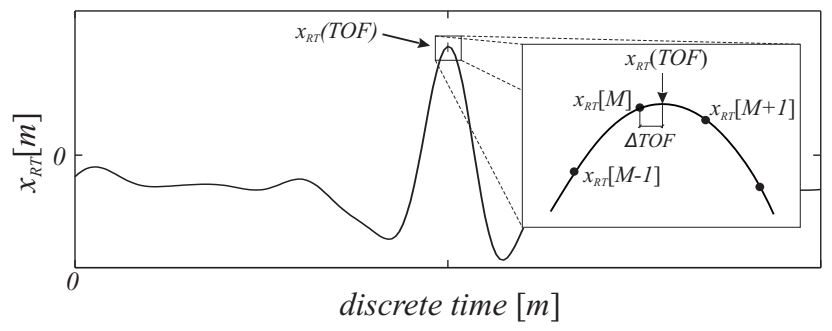

Figure 4: Example of time of flight estimation in discrete time domain. Insert: Detail around the peak of the cross-correlation. 

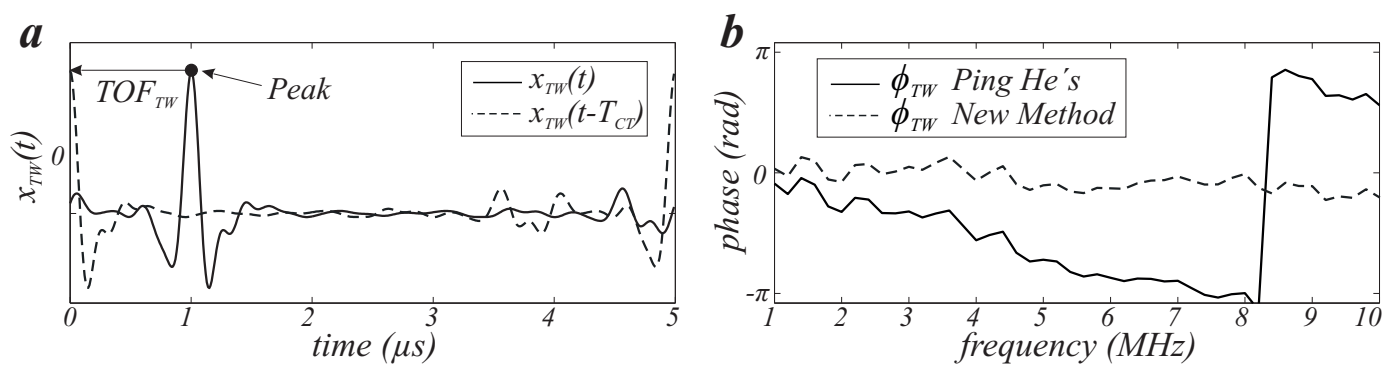

Figure 5: Example for through-transmission parameters calculation using cross-correlation. (a) Crosscorrelation between water-path and through-transmission signals in time domain. (b) Comparison of resulting phase differences using Ping He's method (solid line) and new method (gray stripped line).
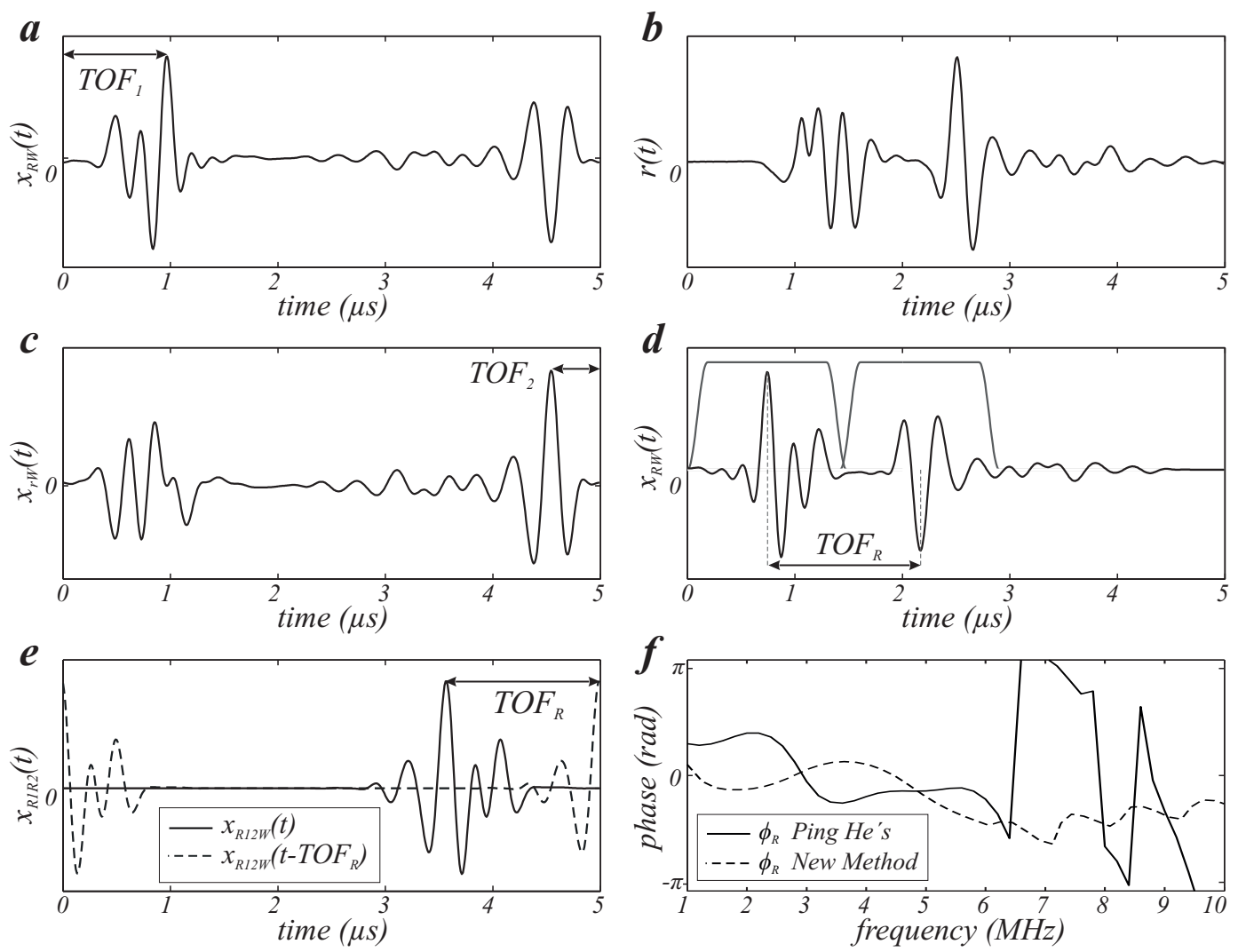

Figure 6: Example iterative deconvolution operation. (a) Cross-Correlation between water-path (Fig. 3a) and pulse-echo (Fig. 3c) signals. (b) Remainder after first iteration. (c) Cross-correlation between remainder and water-path. (d) Cross-correlation and gating windows. (e) Cross-correlation between gated cross-correlations. (f) Resulting phase spectrum using Ping He's (solid line) and the new method (stripped line). 


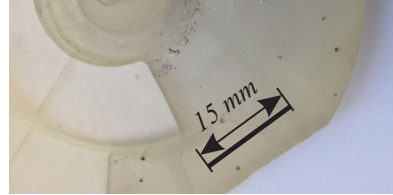

Front view CCE

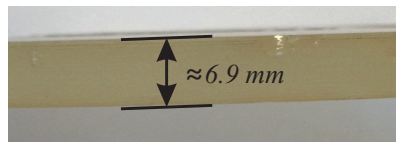

Section view CCE

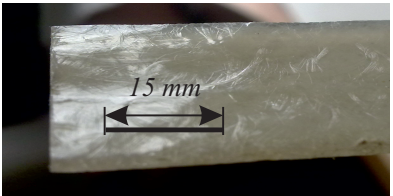

Front view FGRP

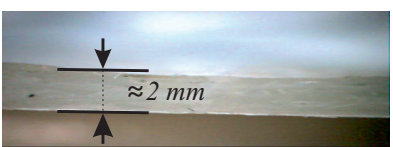

Section view FGRP

Figure 7: Analyzed specimens: CCE front and section views (left). FGRP front and section views.
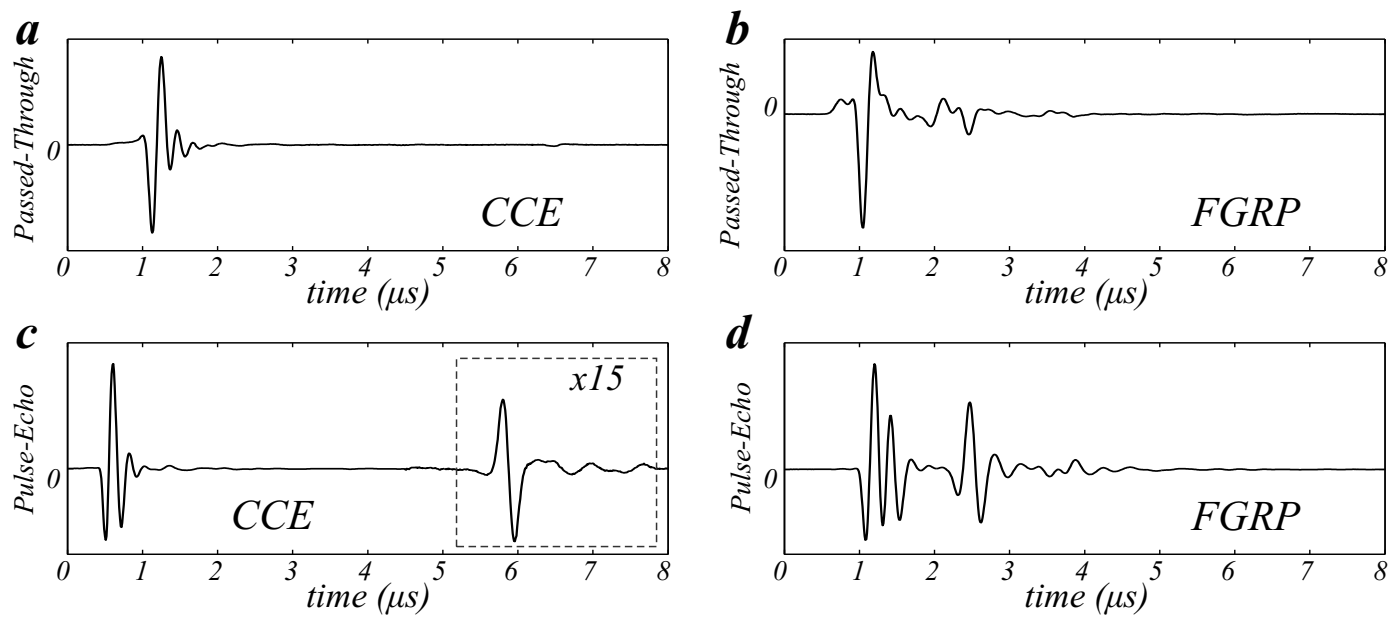

Figure 8: Example of A-scans. (a) CCE passed-through. (b) FGRP passed-through. (c) CCE pulse-echo. (c) FGRP pulse-echo.
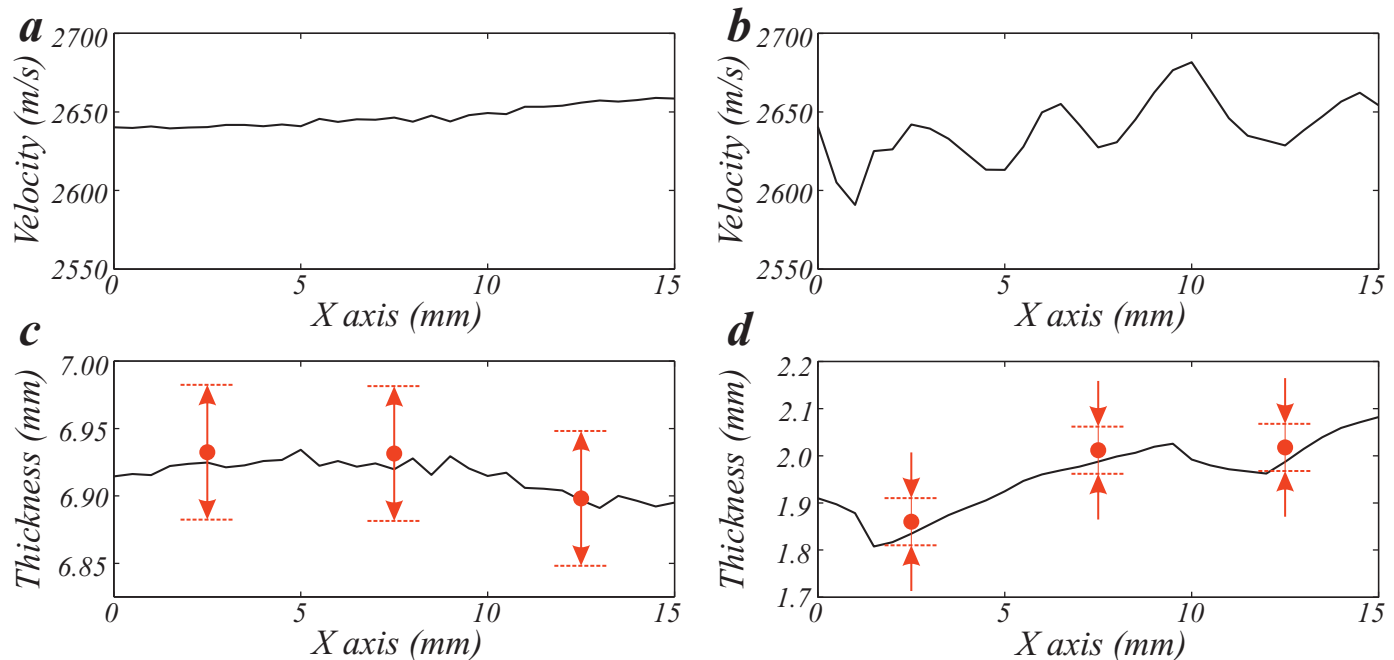

Figure 9: Speed of sound and thickness.(a) Speed of sound for CCE (b) Thickness for CCE (c) Speed of sound for FGRP (d) Thickness for FGRP. 

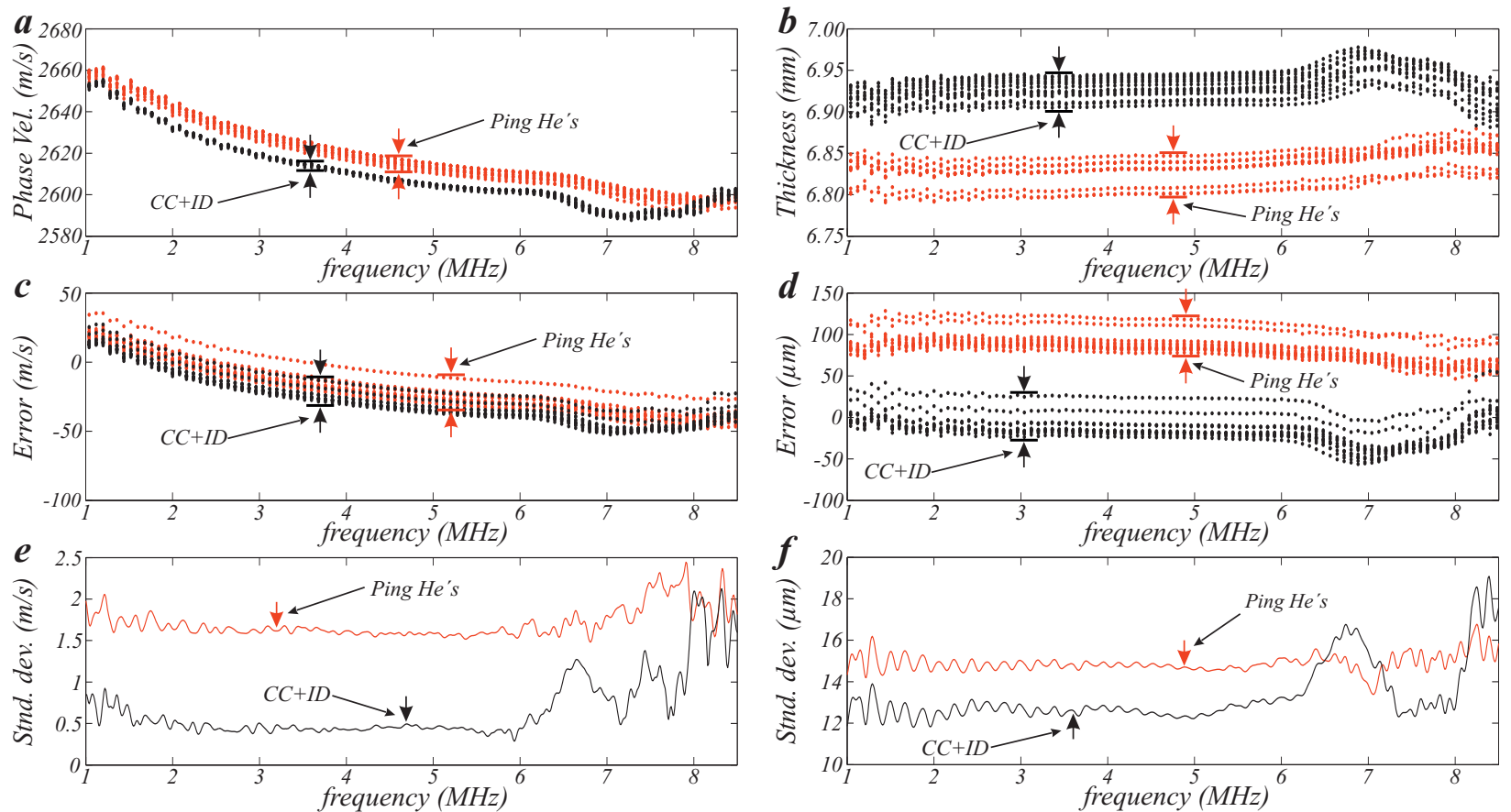

Figure 10: Comparison of phase velocity and thickness for the CCE specimen obtained using Ping He's method (red) and the new procedure (black). (a) Phase velocity in m/s (c) Bias in m/s (e) Standard deviation in $\mathrm{m} / \mathrm{s}$. (b) Thickness in $\mathrm{mm}$ (d) Bias in $\mu m$ (f) Standard deviation in $\mu m$.
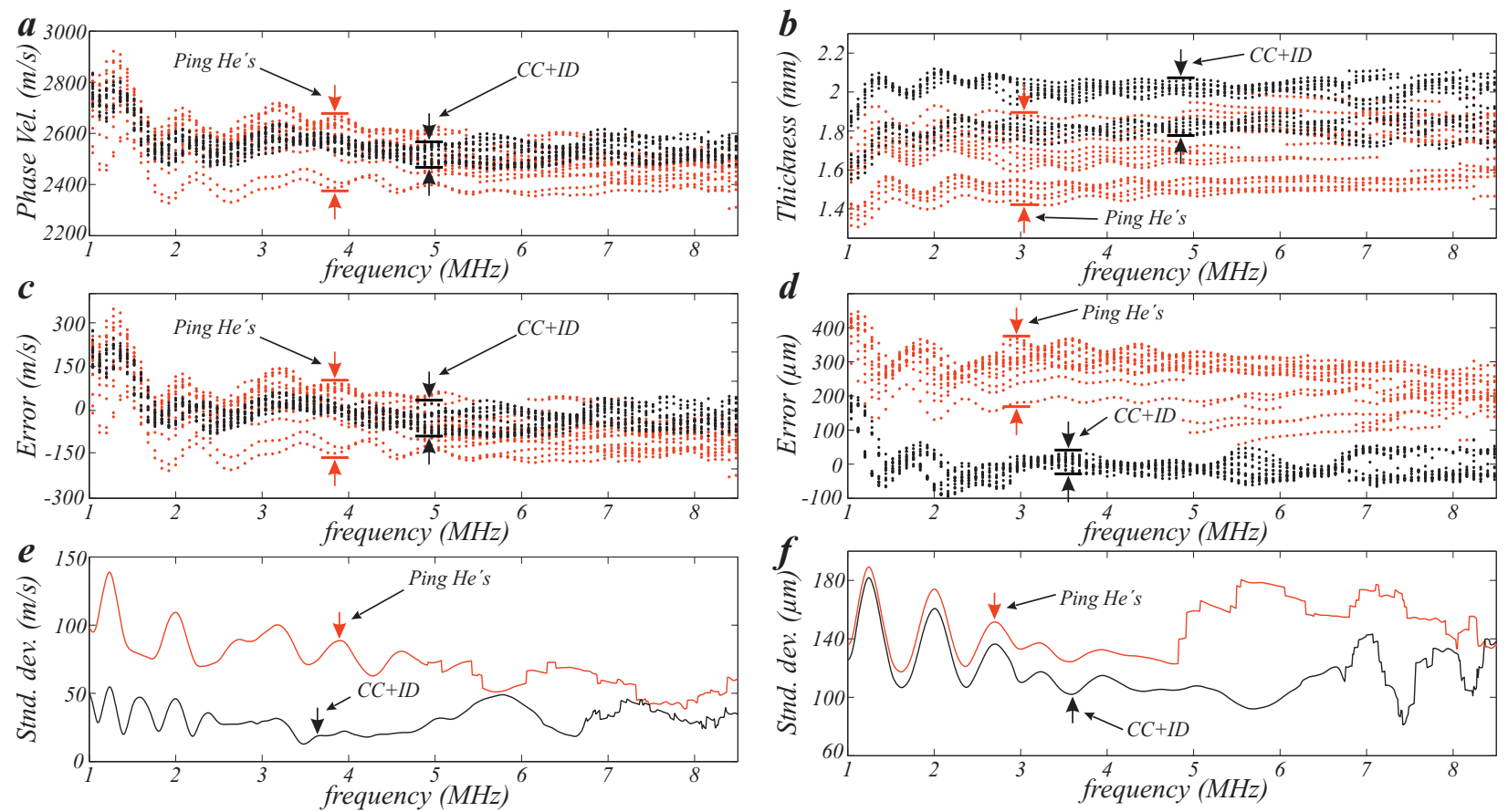

Figure 11: Comparison of phase velocity and thickness for the FGRP specimen obtained using Ping He's method (red) and the new procedure (black). (a) Phase velocity in $\mathrm{m} / \mathrm{s}$ (c) Bias in $\mathrm{m} / \mathrm{s}$ (e) Standard deviation in $\mathrm{m} / \mathrm{s}$. (b) Thickness in mm (d) Bias in $\mu m$ (f) Standard deviation in $\mu m$. 